\title{
SIMULADOR DE UNA PLANTA EXPERIMENTAL DE REFRIGERACIÓN CON UN SISTEMA DE ALMACENAMIENTO DE ENERGÍA
}

\author{
David Rodríguez, Guillermo Bejarano, José A. Alfaya, Manuel G. Ortega \\ Dpto. Ingeniería de Sistemas y Automática, Univ. de Sevilla, \{drgarcia, gbejarano, jalonso9, mortega\}@us.es \\ João M. Lemos \\ INESC-ID, Instituto Superior Técnico, Univ. de Lisboa (Portugal), jlml@inesc-id.pt
}

\section{Resumen}

Este artículo describe el funcionamiento del simulador de una planta experimental de refrigeración por compresión de vapor a la que se añade un sistema de almacenamiento de energía frigorífica. Se aborda el modelado individual de los elementos y su interconexión. Asimismo, se analizan los rangos de potencia frigorífica generados en diferentes modos de operación del sistema, estudiando la interacción entre las distintas potencias implicadas y las limitaciones que se derivan de cara al control.

Palabras clave: Ciclo de refrigeración, Almacenamiento de energía térmica, Material de cambio de fase.

\section{INTRODUCCIÓN}

Cada vez más, la conciencia por reducir las emisiones al medio ambiente toma fuerza. Uno de los grandes factores influyentes, no solo respecto al medio ambiente sino también a nivel socioeconómico, es el gasto energético en producción de frío tanto a nivel industrial [14], como a nivel doméstico [15]. Ante la ingente tarea de reducir el consumo de energía, que atrae cada vez más interés, se plantean nuevos métodos donde, teniendo en cuenta la variación del precio de la potencia eléctrica a lo largo del día, así como la eficiencia variable de los ciclos de refrigeración, los sistemas de almacenamiento de energía térmica ganan atractivo en cuanto a la satisfacción óptima de la demanda frigorífica.

En el Departamento de Ingeniería de Sistemas y Automática de la Universidad de Sevilla se dispone de una planta experimental de refrigeración por compresión de vapor, descrita en [1], la cual cuenta con dos etapas de compresión de distinta potencia, un condensador de aire, y dos recintos a refrigerar con un evaporador y una válvula de expansión por recinto, de forma que se puede operar con temperaturas distintas en cada uno de los recintos. Además, puede ser configurada, bien como un ciclo básico con una etapa de compresión y un recinto, bien como un ciclo con dos etapas y un recinto, o bien como un ciclo con dos etapas y dos recintos. Cada recinto consiste en un depósito, que actúa de almacén de una solución acuosa de glicol; esta es impulsada por una bomba hasta el evaporador, donde se enfría y vuelve al depósito, formando un circuito cerrado. Además, cada depósito cuenta con una resistencia eléctrica variable de hasta $2 \mathrm{~kW}$, que permite simular la carga térmica del recinto a refrigerar.

Siguiendo una reciente línea de investigación en la literatura, se tiene en marcha una ampliación de la planta experimental, incluyendo un sistema de almacenamiento de energía térmica (TES, del inglés Thermal Energy Storage) por recinto, pensado para almacenar excesos de energía frigorífica generados por el ciclo, y cederla cuando se considere necesario al recinto. La Figura 1 muestra la incorporación del sistema TES a un ciclo básico de una etapa y un recinto, donde el tanque TES se dispone en paralelo al evaporador. La incorporación de sistemas TES a los ciclos de refrigeración permite flexibilizar las condiciones de operación, el diseño de equipos más pequeños (evitando el sobredimensionamiento para satisfacer picos de demanda [11]) y un uso más eficiente de los equipos, así como la reducción del consumo de energía [10].

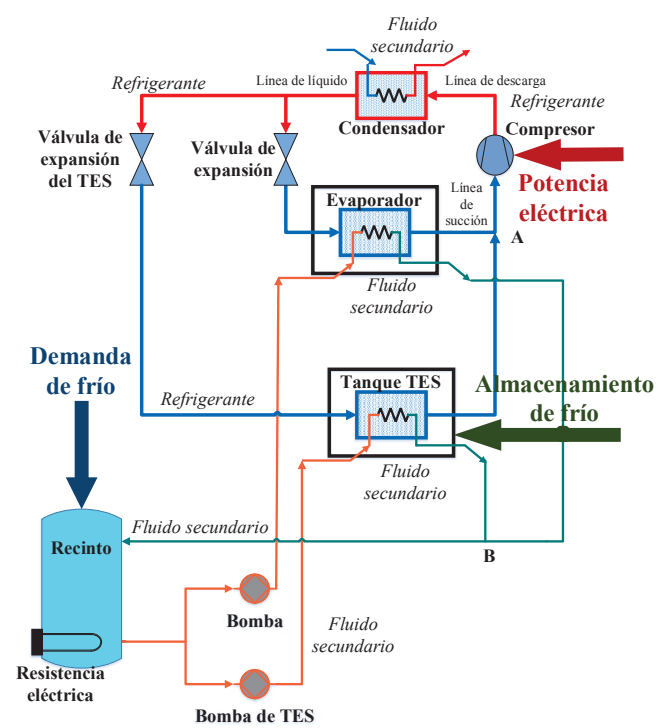

Figura 1: Esquema del ciclo básico de refrigeración ampliado con un sistema TES. 
En este artículo se presenta un simulador dinámico de un ciclo de refrigeración de una etapa de compresión y un único recinto a refrigerar, al que se acopla un sistema de almacenamiento de energía frigorífica. Este simulador, que recoge tanto la dinámica rápida asociada al ciclo de refrigeración, estudiada en [2], como la dinámica más lenta asociada a la carga y descarga del propio tanque TES $[4,5]$, constituye una plataforma que permite plantear y probar estrategias de control sobre este novedoso sistema de forma fácil, rápida y segura. Se presentan los modelos utilizados para cada uno de los elementos del sistema, así como las conexiones entre el ciclo de refrigeración, el tanque TES y el depósito que simula el recinto a refrigerar. Se definen las variables de entrada y salida del simulador completo y se analizan los rangos de funcionamiento de las potencias frigoríficas implicadas, así como la interacción entre las mismas.

El artículo se organiza de la siguiente forma: en la Sección 2 se dan detalles sobre el modelado del ciclo de refrigeración elemento a elemento, se describe el modelo térmico del recinto a refrigerar y el modelo del sistema TES. La Sección 3, tomando el sistema ya como un único bloque, se dedica a describir las variables de entrada y salida, así como sus rangos de funcionamiento, especialmente la interacción entre las potencias frigoríficas implicadas en el ciclo. Finalmente, en la Sección 4 se resumen las principales conclusiones y se proponen algunos trabajos futuros.

\section{MODELADO DEL SISTEMA}

Como se muestra en la Figura 1, el sistema consta de un ciclo básico de refrigeración con una etapa de compresión, un recinto que se pretende refrigerar y un sistema TES. En esta sección se dan algunos detalles del modelado de cada uno de los elementos que componen el sistema.

\subsection{CICLO DE REFRIGERACIÓN}

Un ciclo de refrigeración por compresión de vapor consiste en extraer energía en forma de calor de un foco frío y cederla a un foco caliente, normalmente el ambiente u otro fluido que absorba dicho calor. Se cuenta con dos variables manipulables: la velocidad de giro del compresor $N$ y la apertura de la válvula asociada al evaporador $A_{v}$.

Dado que todo ciclo de refrigeración cuenta al menos con cuatro elementos (evaporador, compresor, condensador y válvula de expansión), cada elemento se ha modelado individualmente para después combinarlos de acuerdo con las interconexiones indicadas en la Figura 1. Además, puesto que los elementos programados usan modelos genéri- cos, es necesario un proceso de identificación de la planta a simular, para definir ciertos parámetros. En la literatura asociada se detallan los modelos y el procedimiento de identificación de la planta experimental $[3,12]$.

\subsubsection{VÁLVULA DE EXPANSIÓN}

La válvula de expansión, basada en el modelo propuesto en [13], permite calcular el caudal circulante $\dot{m}$ por medio de (1). Dado el coeficiente de apertura de la válvula de expansión $C_{e e v}$ (relacionado con su geometría), la apertura de válvula $A_{v}$, la diferencia de presión entre su entrada $P_{c, \text { out }}$ y su salida $P_{e}$, y la densidad a la entrada $\rho_{c, \text { out }}$ (calculada indirectamente mediante la entalpía $\left.h_{c, o u t}\right)$, se calcula el caudal de refrigerante $\dot{m}$.

$$
\dot{m}=C_{\text {eev }} A_{v} \sqrt{2 \rho_{c, \text { out }}\left(P_{c, \text { out }}-P_{e}\right)}
$$

\subsubsection{COMPRESOR}

El compresor, basado en el modelo propuesto en [13], conocido ciertos parámetros ligados al material y geometría, recibe como entradas la velocidad de giro $N$, la presión de descarga $P_{c, i n}$, el caudal circulante $\dot{m}$, la entalpía a la entrada $h_{c o m p, i n} \mathrm{y}$ la temperatura ambiente $T_{\text {surr }}$. Como salidas se tienen la presión de succión $P_{e}$, la entalpía de salida $h_{c, i n}$ y la potencia mecánica consumida $\dot{W}_{c o m p}$. Para ello, se tiene el conjunto (2), que se ejecuta iterativamente, ya que algunos valores intermedios son calculados a partir de $P_{e}$, hasta que la presión propuesta y la resultante convergen.

$$
\begin{aligned}
& \dot{m}=S_{t}\left[1-c\left(\left(\frac{P_{c, i n}}{P_{e}}\right)^{\frac{c_{v, e, g}}{c_{p, e, g}}}-1\right)\right] \frac{N}{v_{e}} \\
& \dot{W}_{c o m p}=b \dot{m}\left(h_{c, i n, i s}-h_{c o m p, i n}\right) \\
& T_{c, i n, i s}=T_{c}+\frac{h_{c, i n, i s}-h_{c, g}}{c_{p, c, g}} \\
& h_{c, i n}=h_{c o m p, i n}+\frac{\dot{W}_{c o m p}-U A\left(T_{c, i n, i s}-T_{s u r r}\right)}{\dot{m}}
\end{aligned}
$$

\subsubsection{EVAPORADOR}

El modelo del evaporador se explica con más detalle en $[3,12]$, mientras que su diagrama de temperaturas se muestra en la Figura 2. El intercambiador se divide en dos zonas de operación: zona bifásica y zona de vapor sobrecalentado, y el modelo cuenta con dos modos de operación, véase la Figura 3, dependiendo de la entalpía del refrigerante a la salida en relación a la de vapor saturado. Conocidos parámetros del material, geometría y coeficientes de transmisión de calor en cada zona 
entre el refrigerante y el fluido secundario $\left(\alpha_{e, t p} \mathrm{y}\right.$ $\left.\alpha_{e, s h}\right)$, el modelo recibe como entradas la entalpía $h_{e, i n}$, presión $P_{e}$ y caudal másico $\dot{m}_{e}$ del refrigerante a la entrada, además de valores de temperatura, caudal y presión del fluido secundario a la entrada $\left(T_{e, s e c, i n}, \dot{m}_{e, s e c}\right.$ y $\left.P_{e, s e c}\right)$. Las salidas de mayor interés son la potencia frigorífica transferida en las zonas bifásica $\dot{Q}_{e, t p}$ y sobrecalentada $\dot{Q}_{e, s h}$, la temperatura de salida del fluido secundario $T_{e, s e c, o u t}$, la entalpía del refrigerante a la salida $h_{e, \text { out }}$ y el grado de sobrecalentamiento $\left(T_{S H}\right)$, que se define como la diferencia entre la temperatura del refrigerante a la salida del evaporador y la temperatura de saturación a la presión de evaporación $P_{e}$. Se utiliza el método $\epsilon-N T U$ [8] en un proceso iterativo, de forma que, por medio de los coeficientes $\alpha_{e, t p}$ y $\alpha_{e, s h}$, se obtienen los valores de $\dot{Q}_{e, t p}$ y $\dot{Q}_{e, s h}$, que permiten la obtención del resto de variables de salida. La potencia frigorífica total transferida en el evaporador $\dot{Q}_{e}$ es la suma de las potencias parciales $\dot{Q}_{e, t p}$ y $\dot{Q}_{e, s h}$.

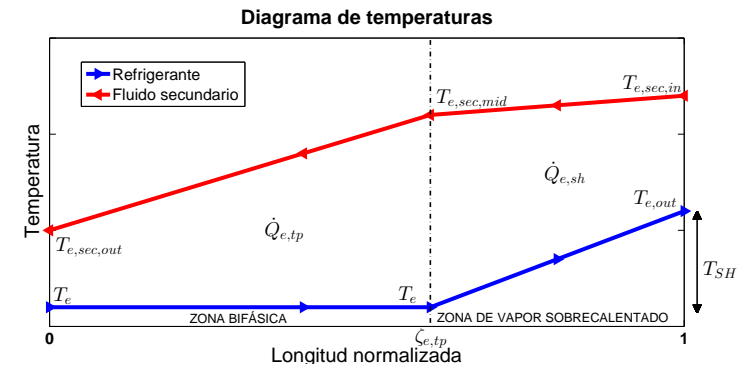

Figura 2: Diagrama de temperaturas en el evaporador.

\section{EVAPORADOR}

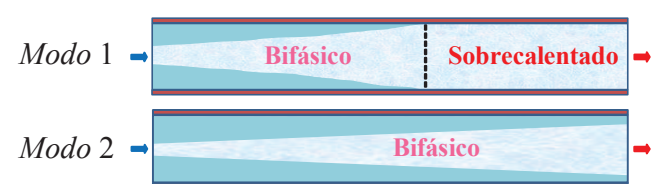

Figura 3: Modos de operación del evaporador.

\subsubsection{CONDENSADOR}

El condensador, cuyo modelo se detalla en $[3,6$, 12], es el único bloque dinámico del ciclo de refrigeración, debido a que bajo ciertas circunstancias su dinámica resulta dominante, pudiéndose considerar el resto de elementos como estáticos [2]. El diagrama de temperaturas y presiones a lo largo del condensador se muestra en la Figura 4. Del mismo modo que en el evaporador, se divide el intercambiador en tres zonas: zona de vapor sobrecalentado, zona bifásica y zona de líquido subenfriado, y atendiendo a la entalpía específica de salida y entrada del refrigerante se distinguen cinco modos de operación, véase la Figura 5. Conocidos valores relativos al material, geometría y coeficientes de transmisión de calor en cada zona entre el refrigerante y el fluido secundario $\left(\alpha_{c, s c}, \alpha_{c, t p}\right.$ y $\left.\alpha_{c, s h}\right)$, el modelo recibe como entradas la entalpía $h_{c, i n}$, presión $P_{c, i n}$ y caudal másico $\dot{m}$ del refrigerante a la entrada, además de valores de temperatura, caudal y presión del fluido secundario a la entrada $\left(T_{c, s e c, i n}, \dot{m}_{c, s e c}\right.$ y $\left.P_{c, s e c}\right)$. Las variables de salida más relevantes son la entalpía $h_{c, \text { out }}$ y presión $P_{c, \text { out }}$ del refrigerante a la salida, pudiendo ser esta última diferente a la de entrada $P_{c, \text { in }}$ debido a la pérdida de carga asociada. Se aplica de nuevo el método $\epsilon-N T U$ [8] en un proceso iterativo.

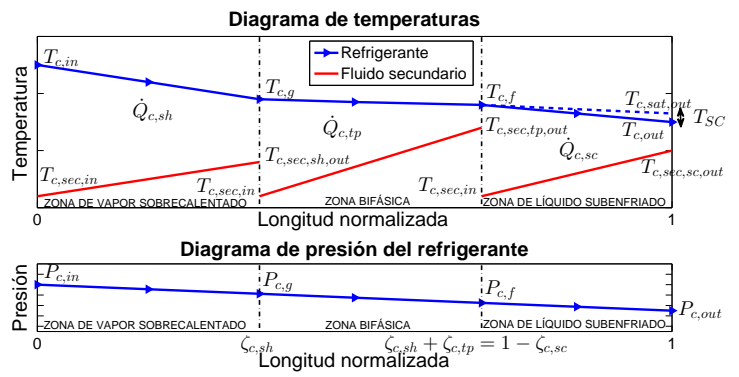

Figura 4: Diagrama de presiones y temperaturas en el condensador.

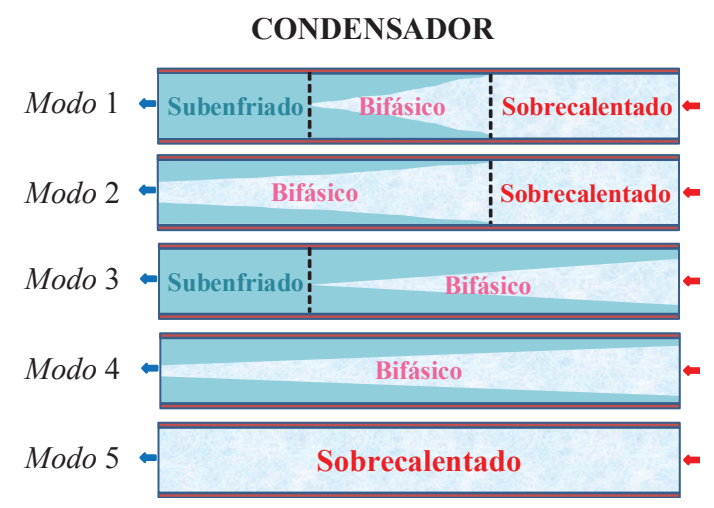

Figura 5: Modos del operación del condensador.

\subsection{RECINTO A REFRIGERAR}

El recinto se simula mediante un depósito que almacena una solución acuosa de glicol propulsada por bombas en un circuito cerrado. Esta solución acuosa puede circular a través del evaporador como fluido secundario y/o a través del tanque TES, como se muestra en la Figura 1. El modelo térmico responde a (3), donde se realiza un balance de potencia incluyendo las potencias frigoríficas recibidas en el evaporador $\dot{Q}_{e}$ y en el tanque TES $\dot{Q}_{T E S, s e c}$, el aporte externo de calor $\dot{Q}_{R}$ generado por la resistencia eléctrica, y las pérdidas térmicas con el ambiente mediante el coeficiente de perdidas térmicas $\alpha_{\text {tank }}$, dando como resultado la evolución de la temperatura del depósito $T_{\text {tank }}$. 


$$
\begin{aligned}
& \rho_{\text {sec }} c_{p, s e c} V_{\text {tank }} \frac{d T_{\text {tank }}}{d t}= \\
& =\dot{Q}_{R}-\dot{Q}_{e}-\dot{Q}_{T E S, s e c}-\alpha_{\text {tank }}\left(T_{\text {tank }}-T_{\text {surr }}\right)
\end{aligned}
$$

Además, debido a que las bombas que propulsan el fluido secundario se consideran ideales, se tiene que la temperatura de entrada del fluido secundario en el evaporador $T_{e, s e c, i n} \mathrm{y}$ la temperatura de entrada del fluido secundario en el TES $T_{T E S, \text { sec, in }}$ coinciden con la temperatura del depósito $T_{\text {tank }}$.

\subsection{SISTEMA DE ALMACENAMIENTO DE ENERGÍA TÉRMICA}

Los sistemas de almacenamiento de energía térmica de calor latente LHTES son aquellos que aprovechan como método de almacenamiento el cambio de fase de un material, ya sea tanto para calor (vía fusión) como para frío (vía solidificación) [9]. Cualquier sistema LHTES para almacenamiento de frío debe estar compuesto como mínimo por:

- Una sustancia de almacenamiento, denominada PCM (del inglés Phase Change Material), la cual, mediante cambio de fase y manteniendo aproximadamente constante su temperatura, permite almacenar energía frigorífica en forma de frío latente.

- Un recipiente contenedor para el PCM.

- Una superficie de intercambio de calor entre el PCM y el fluido a refrigerar o foco caliente, y entre el PCM y el fluido refrigerante o foco frío.

El sistema físico a construir se ha diseñado, véase la Figura 6, como un tanque cilíndrico, con 17 tubos destinados a contener el PCM, 36 tubos que atraviesan el tanque y transportan el refrigerante, y 32 tubos que atraviesan el tanque y transportan la solución acuosa de glicol procedente del depósito. Además, otra solución acuosa de glicol, denominado fluido intermedio, llena el volumen sobrante en el tanque. Se trata de un fluido de una alta conductividad térmica que actúa de canal para la transmisión de potencia frigorífica entre los tubos de PCM y los sumideros (solución de glicol) o fuentes (refrigerante) de potencia frigorífica.

El modelo de simulación del sistema TES, que se inspira en [5], recibe como entradas, además de parámetros relacionados con su geometría y fluidos (solución de glicol, refrigerante, PCM y fluido intermedio): su modo de funcionamiento, información sobre las corrientes de entrada de refrige-

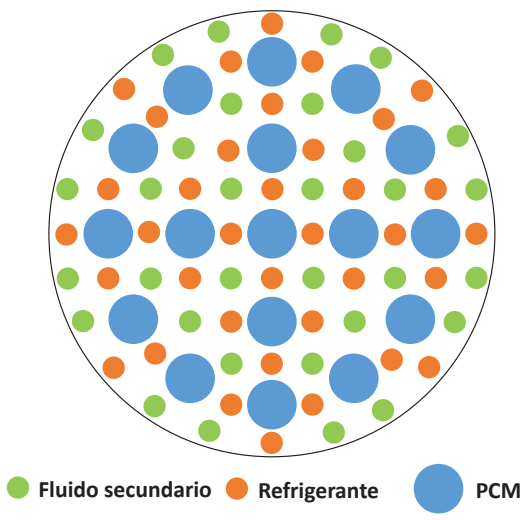

Figura 6: Vista en planta del tanque TES.

rante y fluido secundario, y la temperatura ambiente. Se distinguen cuatro modos de funcionamiento del tanque TES, relacionados con la existencia, o no, de caudales efectivos de refrigerante y fluido secundario. El modo de carga es aquel en que solo circula refrigerante $\left(\dot{Q}_{T E S, \text { refr }}>0\right)$, mientras que en el modo de descarga solo circula fluido secundario $\left(\dot{Q}_{T E S, s e c}>0\right)$. En el modo combinado de carga y descarga ambos fluidos circulan $\left(\dot{Q}_{T E S, r e f r}>0, \dot{Q}_{T E S, \text { sec }}>0\right)$, mientras que en el modo reposo no circula ninguno de los dos $\left(\dot{Q}_{T E S, r e f r}=0, \dot{Q}_{T E S, s e c}=0\right)$.

Los caudales másicos de ambos fluidos $\left(\dot{m}_{T E S, r e f r}\right.$ y $\left.\dot{m}_{T E S, s e c}\right)$ representan las variables manipulables. En el caso del refrigerante se ha añadido el modelo de la válvula de expansión correspondiente, lo que implica que la actuación no se realiza directamente sobre el caudal $\dot{m}_{T E S, \text { refr }}$, sino sobre la apertura de válvula $A_{v, T E S}$, mientras que se consideran su presión $P_{e}$ y entalpía específica $h_{c, \text { out }}$ a la entrada del TES para caracterizar su estado termodinámico. En el caso del fluido secundario, se caracteriza su estado termodinámico mediante su presión $P_{T E S, s e c}$ y temperatura $T_{T E S, s e c}$ a la entrada del TES, pero su caudal másico $\dot{m}_{T E S, \text { sec }}$ se manipula directamente, lo que resultaría en la práctica en una referencia para el control de bajo nivel de la bomba correspondiente.

El modelo proporciona información detallada del estado del PCM, fluido intermedio, refrigerante y fluido secundario, siendo las variables de mayor interés la entalpía de salida del refrigerante $h_{T E S, \text { out }}$, la temperatura del fluido intermedio $T_{T E S, i n t}$ y las potencias frigoríficas absorbida $\dot{Q}_{T E S, r e f r}$ y cedida $\dot{Q}_{T E S T, \text { sec }}$ por parte del PCM.

La principal variable de estado del modelo es el estado de carga del PCM, además de la temperatura del fluido intermedio $T_{T E S, i n t}$. Se considera un modelo discretizado de los cilindros de PCM, donde se divide el volumen total de cada cilindro en 


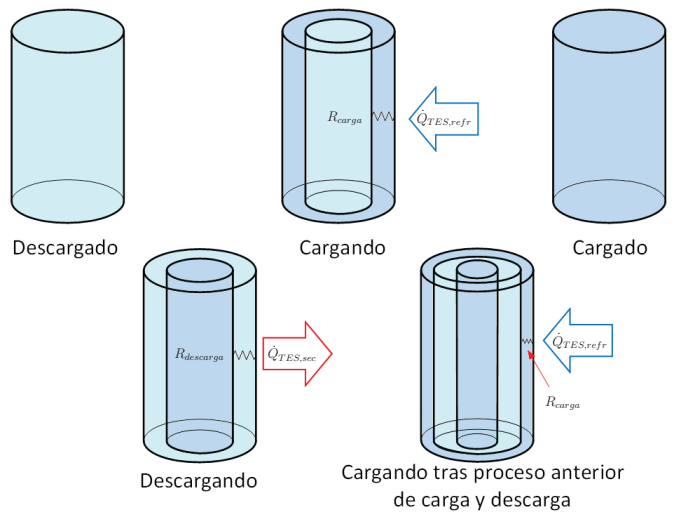

Figura 7: Modelo radial de los cilindros de PCM.

una serie de capas radiales, véase la Figura 7, cuyo estados entálpicos representan variables de estado. Las capas se van solidificando (carga de frío) o fundiendo (descarga de frío), de una en una y desde la periferia hacia el interior del cilindro, ya que este intercambia calor con el fluido intermedio. En cada instante de tiempo se calcula la posición del frente de fusión o solidificación, que estará localizado en una capa determinada con entalpía específica entre los límites de latencia. Las capas exteriores se encontrarán en zona sensible, mientras que las interiores aún estarán en zona latente. Se estima la energía transferida con el fluido intermedio y se evalúa si la capa donde se sitúa el frente de fusión/solidificación agotará su energía latente: si es así, el frente se mueve a la capa inmediatamente interior, y se repite el proceso con el exceso de energía; si no, el frente permanecerá en dicha capa, que simplemente modifica su estado entálpico dentro de los límites de latencia. Las potencias transferidas $\dot{Q}_{T E S, r e f r}$ y/o $\dot{Q}_{T E S, s e c}$ varían en función de la posición del frente de fusión/solidificación, ya que las capas exteriores en zona sensible actúan a modo de resistencia térmica $\left(R_{\text {carga }}\right.$ o $R_{\text {descarga }}$, tal como se muestra en la Figura 7). El estado de carga global del PCM se calcula a partir de las entalpías específicas de todas las capas radiales.

\section{SIMULADOR DEL SISTEMA}

\subsection{ESTRUCTURA Y RANGOS DE ENTRADA}

El simulador se compone, como se muestra en la Figura 8, de un único bloque que incluye el ciclo de refrigeración y el tanque TES. Como entradas, se tienen cuatro variables manipulables: velocidad de giro del compresor $N$, aperturas de las válvulas asociadas al evaporador $A_{v} \mathrm{y}$ al TES $A_{v, T E S}$, y caudal de fluido secundario del TES $\dot{m}_{T E S, s e c}$. Además, también se reciben datos de las perturba- ciones del sistema: caudales másicos, temperaturas de entrada y presiones de los distintos fluidos secundarios y la temperatura ambiente. Como salidas, aunque se tiene disponible toda la información de salida de cada elemento, se han considerado el grado de sobrecalentamiento a la entrada del compresor $T_{S H}$, ya que en sistemas reales se debe garantizar un valor mínimo de $T_{S H}$ que impida la entrada de gotas de líquido en el compresor, la potencia frigorífica transferida en el evaporador $\dot{Q}_{e}$, las potencias transferidas en el TES por parte del refrigerante $\dot{Q}_{T E S, r e f r}$ y del fluido secundario $\dot{Q}_{T E S, \text { sec }}$ y el coeficiente de comportamiento COP del ciclo ampliado.

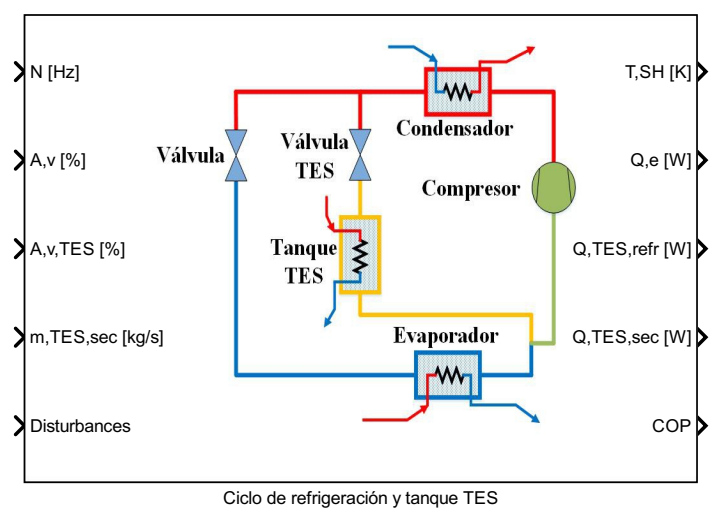

Figura 8: Simulador del ciclo de refrigeración ampliado con el tanque TES.

El simulador ha sido programado bajo el entorno de Simulink ${ }^{\circledR}$, MATLAB $^{\circledR}$ R2014a, Windows ${ }^{\circledR} 7$, 64 bit. Además, para conocer las propiedades termodinámicas de los fluidos que intervienen, se ha hecho uso de la librería Coolprop [7].

Para trabajar con el simulador se necesita una inicialización previa a la ejecución que garantice un punto inicial admisible y de equilibrio, o en caso de no ser posible o no desearse realizar el proceso de inicialización, se cuenta con valores iniciales precalculados, aunque estos provocan un transitorio inicial de ajuste. En la Tabla 1 se recoge el rango de funcionamiento de las variables de entrada.

\subsection{INTERACCIÓN ENTRE POTENCIAS FRIGORÍFICAS}

En base a los rangos de las variables de entrada, se ha obtenido el rango de las potencias de salida, para lo que se ha definido un conjunto de modos globales de operación del sistema, véase la Figura 9 , relacionados con la interacción y actuación de las potencias $\dot{Q}_{e}, \dot{Q}_{T E S, r e f r}$ y $\dot{Q}_{T E S, \text { sec }}$. Además, se ha diseñado un control de caudal másico de refrigerante, tanto del evaporador $\dot{m}_{e}$ como del TES $\dot{m}_{T E S, r e f r}$, actuando por medio de sus respectivas válvulas de expansión $A_{v}$ y $A_{v, T E S}$, tratan- 


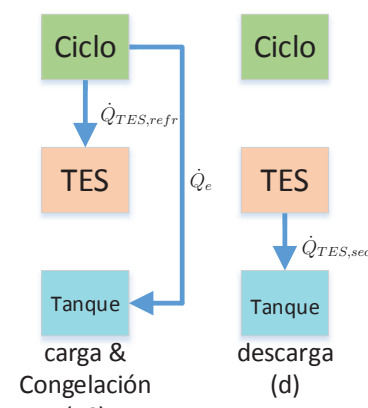

(cC)

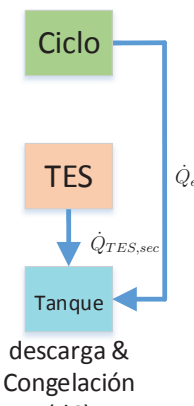

(dC)

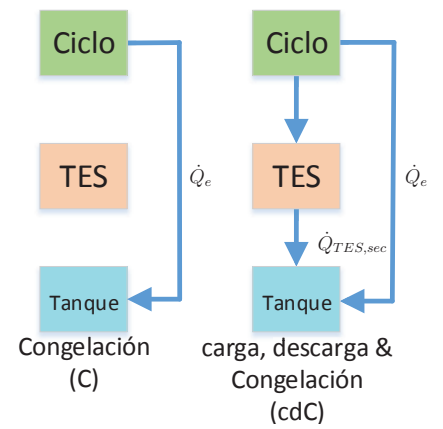

(cdC)

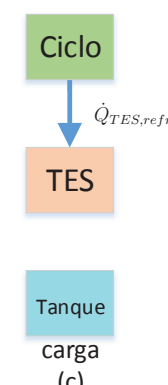

(c)

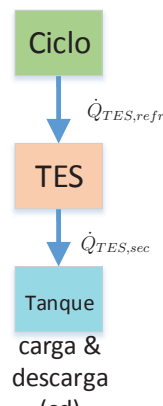

(cd)
Ciclo

Reposo

$(-)$

Figura 9: Modos globales de operación del sistema.

Tabla 1: Rangos de entradas del simulador.

\begin{tabular}{|c|c|c|c|}
\hline Variable & Símbolo & Rango & Unidad \\
\hline \multicolumn{4}{|c|}{ Variables manipulables } \\
\hline $\begin{array}{l}\text { Velocidad de giro del } \\
\text { compresor }\end{array}$ & $N$ & $0 \cup[30,50]$ & $\mathrm{Hz}$ \\
\hline $\begin{array}{l}\text { Apertura de la válvula } \\
\text { asociada al evaporador }\end{array}$ & $A_{v}$ & {$[0,100]$} & $\%$ \\
\hline $\begin{array}{l}\text { Apertura de la válvula } \\
\text { asociada al TES }\end{array}$ & $A_{v, T E S}$ & {$[0,100]$} & $\%$ \\
\hline $\begin{array}{l}\text { Caudal másico del fluido } \\
\text { secundario del TES }\end{array}$ & $\dot{m}_{T E S, s e c}$ & {$[0,0.4]$} & $\mathrm{kg} \mathrm{s}^{-1}$ \\
\hline \multicolumn{4}{|c|}{ Perturbaciones } \\
\hline $\begin{array}{c}\text { Temperatura de entrada } \\
\text { del fluido secundario del } \\
\text { condensador }\end{array}$ & $T_{c, s e c, i n}$ & {$[27,33]$} & ${ }^{\circ} \mathrm{C}$ \\
\hline $\begin{array}{l}\text { Caudal másico del fluido } \\
\text { secundario del } \\
\text { condensador }\end{array}$ & $\dot{m}_{c, s e c}$ & {$[0.125,0.175]$} & $\mathrm{kg} \mathrm{s}^{-1}$ \\
\hline $\begin{array}{l}\text { Presión del fluido } \\
\text { secundario del } \\
\text { condensador }\end{array}$ & $P_{c, s e c}$ & {$[0.5,2]$} & bar \\
\hline $\begin{array}{l}\text { Temperatura de entrada } \\
\text { del fluido secundario del } \\
\text { evaporador }\end{array}$ & $T_{e, s e c, i n}$ & {$[-22,-18]$} & ${ }^{\circ} \mathrm{C}$ \\
\hline $\begin{array}{l}\text { Caudal másico del fluido } \\
\text { secundario del evaporador }\end{array}$ & $\dot{m}_{e, s e c}$ & {$[0.055,0.075]$} & $\mathrm{kg} \mathrm{s}^{-1}$ \\
\hline $\begin{array}{l}\text { Presión del fluido } \\
\text { secundario del evaporador }\end{array}$ & $P_{e, s e c}$ & {$[0.5,2]$} & bar \\
\hline Temperatura ambiente & $T_{\text {surr }}$ & {$[20,30]$} & ${ }^{\circ} \mathrm{C}$ \\
\hline $\begin{array}{l}\text { Potencia de la resistencia } \\
\text { eléctrica del depósito }\end{array}$ & $\dot{Q}_{R}$ & {$[0,2000]$} & $\mathrm{W}$ \\
\hline $\begin{array}{l}\text { Temperatura de entrada } \\
\text { del fluido secundario del } \\
\text { TES }\end{array}$ & $T_{T E S, s e c, i n}$ & {$[-22,-18]$} & ${ }^{\circ} \mathrm{C}$ \\
\hline $\begin{array}{l}\text { Presión del fluido } \\
\text { secundario del TES }\end{array}$ & $P_{T E S, s e c}$ & {$[0.5,2]$} & bar \\
\hline
\end{tabular}

do de evitar la no linealidad de las mismas, y un controlador para $T_{S H}$ por medio de la velocidad del compresor $N$. Ambos caudales másicos $\dot{m}_{e} \mathrm{y}$ $\dot{m}_{T E S, r e f r}$ son controlados por un controlador PI junto con un bloque feedfoward que invierte el modelo de la válvula mostrado en (1), mientras que $T_{S H}$ se controla mediante un regulador PI.

Una vez controlados los caudales másicos y el $T_{S H}$, bajo condiciones nominales de las perturbaciones, recogidas en la Tabla 2, y sin considerar la dinámica del recinto a refrigerar, lo que puede verse como una simulación donde la combinación de la potencia frigorífica aportada al fluido secundario en el evaporador y en el TES sigue a la potencia térmica $\dot{Q}_{R}$ generada por la resistencia eléctrica en el recinto, se determina el rango de potencias del sis- tema. Se consideran todos los modos globales definidos en la Figura 9 y tres diferentes estados de carga del PCM (totalmente cargado, media carga y totalmente descargado). Para ello se realiza un barrido para todo el rango de caudales del sistema, donde la referencia para el grado de sobrecalentamiento $T_{S H}^{r e f}$ fuerza que la velocidad del compresor $N$ sea tan lenta como sea posible siempre que $T_{S H}$ no caiga por debajo de $2^{\circ} \mathrm{C}$. Los resultados obtenidos se recogen en la Tabla 3. Se observa la influencia del estado de carga del TES sobre las potencias obtenidas, principalmente sobre $\dot{Q}_{T E S, s e c}$.

Tabla 2: Valores nominales de las perturbaciones del sistema.

\begin{tabular}{ccc}
\hline Variable & Valor & Unidad \\
\hline$T_{c, \text { sec }, \text { in }}$ & 30 & ${ }^{\circ} \mathrm{C}$ \\
$\dot{m}_{c, \text { sec }}$ & 0.15 & $\mathrm{~kg} \mathrm{~s}^{-1}$ \\
$P_{c, s e c}$ & 1 & $\mathrm{bar}$ \\
$T_{e, s e c, i n}$ & -20 & ${ }^{\circ} \mathrm{C}$ \\
$\dot{m}_{e, s e c}$ & 0.065 & $\mathrm{~kg} \mathrm{~s}^{-1}$ \\
$P_{e, s e c}$ & 1 & $\mathrm{bar}$ \\
$T_{\text {surr }}$ & 25 & ${ }^{\circ} \mathrm{C}$ \\
$\dot{Q}_{R}$ & 500 & $\mathrm{~W}$ \\
$T_{T E S, \text { sec }, \text { in }}$ & -20 & ${ }^{\circ} \mathrm{C}$ \\
$P_{T E S, \text { sec }}$ & 1 & $\mathrm{bar}$ \\
\hline
\end{tabular}

En las Figuras 10 a 12 se muestra la relación cualitativa entre las potencias y los caudales más influyentes para cada una, omitiendo aquellas que pueden ser consideradas despreciables. Se ha seleccionado el modo cdC, donde intervienen las tres potencias, siendo una buena representación global para cualquier modo de operación donde intervengan más de una. Se observa que $\dot{Q}_{e}$ y $\dot{Q}_{T E S, r e f r}$ están acoplados con ambos caudales de refrigerante $\dot{m}_{e}$ y $\dot{m}_{T E S, r e f r}$, mientras que $\dot{Q}_{T E S, \text { sec }}$ se ve afectado por $\dot{m}_{T E S, r e f r}$ y $\dot{m}_{T E S, s e c}$. Además, se observa que los valores extremos de los rangos de potencia pueden no ser alcanzables según el valor de las otras potencias que intervienen.

\section{CONCLUSIONES Y TRABAJOS FUTUROS}

En este trabajo se ha presentado el simulador de una planta experimental de refrigeración a la que 
Tabla 3: Rangos de salidas del sistema.

\begin{tabular}{|c|c|c|c|c|c|c|}
\hline Modo & $\dot{m}_{e}\left[\mathrm{~g} \mathrm{~s}^{-1}\right]$ & $\dot{Q}_{e}[\mathrm{~W}]$ & $\dot{m}_{T E S, r e f r}\left[\mathrm{~g} \mathrm{~s}^{-1}\right]$ & $\dot{Q}_{T E S, r e f r}[\mathrm{~W}]$ & $\dot{m}_{T E S, \sec }\left[\mathrm{g} \mathrm{s}^{-1}\right]$ & $\dot{Q}_{T E S, \sec }[\mathrm{W}]$ \\
\hline \multicolumn{7}{|c|}{ TES totalmente cargado } \\
\hline $\mathrm{cC}$ & {$[2,9]$} & {$[179,698]$} & {$[2,9]$} & {$[165,700]$} & - & - \\
\hline $\mathrm{d}$ & - & - & - & - & {$[50,400]$} & {$[600,1059]$} \\
\hline $\mathrm{dC}$ & {$[2,10]$} & {$[225,807]$} & - & - & {$[50,400]$} & {$[600,1059]$} \\
\hline $\mathrm{C}$ & {$[2,10]$} & {$[225,807]$} & - & - & - & - \\
\hline $\mathrm{cdC}$ & {$[2,9]$} & {$[179,701]$} & {$[2,9]$} & {$[170,730]$} & {$[50,400]$} & {$[657,1262]$} \\
\hline $\mathrm{c}$ & - & - & {$[2,8.6]$} & {$[210,600]$} & - & - \\
\hline $\mathrm{cd}$ & - & - & {$[2,8.6]$} & {$[215,624]$} & {$[50,400]$} & {$[658,1243]$} \\
\hline \multicolumn{7}{|c|}{ TES a media carga } \\
\hline $\mathrm{cC}$ & {$[2,9]$} & {$[179,701]$} & {$[2,9]$} & {$[166,703]$} & - & - \\
\hline $\mathrm{d}$ & - & - & - & - & {$[50,400]$} & {$[531,913]$} \\
\hline $\mathrm{dC}$ & {$[2,10]$} & {$[225,807]$} & - & - & {$[50,400]$} & {$[531,913]$} \\
\hline $\mathrm{C}$ & {$[2,10]$} & {$[225,807]$} & - & - & - & - \\
\hline $\mathrm{cdC}$ & {$[2,9]$} & {$[179,701]$} & {$[2,9]$} & {$[170,732]$} & {$[50,400]$} & {$[622,1195]$} \\
\hline $\mathrm{c}$ & - & - & {$[2,8.6]$} & {$[210,602]$} & - & - \\
\hline $\mathrm{cd}$ & - & - & {$[2,8.6]$} & {$[216,626]$} & {$[50,400]$} & {$[623,1172]$} \\
\hline \multicolumn{7}{|c|}{ TES totalmente descargado } \\
\hline $\mathrm{cC}$ & {$[2,9]$} & {$[180,701]$} & {$[2,9]$} & {$[166,720]$} & - & - \\
\hline $\mathrm{d}$ & - & - & - & - & {$[50,400]$} & {$[468,787]$} \\
\hline $\mathrm{dC}$ & {$[2,10]$} & {$[225,807]$} & - & - & {$[50,400]$} & {$[468,787]$} \\
\hline $\mathrm{C}$ & {$[2,10]$} & {$[225,807]$} & - & - & - & - \\
\hline $\mathrm{cdC}$ & {$[2,9]$} & {$[179,701]$} & {$[2,9]$} & {$[170,734]$} & {$[50,400]$} & {$[588,1132]$} \\
\hline $\mathrm{c}$ & - & - & {$[2,8.6]$} & {$[211,608]$} & - & - \\
\hline $\mathrm{cd}$ & - & - & {$[2,8.6]$} & {$[215,628]$} & {$[50,400]$} & {$[589,1107]$} \\
\hline
\end{tabular}

Potencia frigorífica transferida en el evaporador, Modo cdC

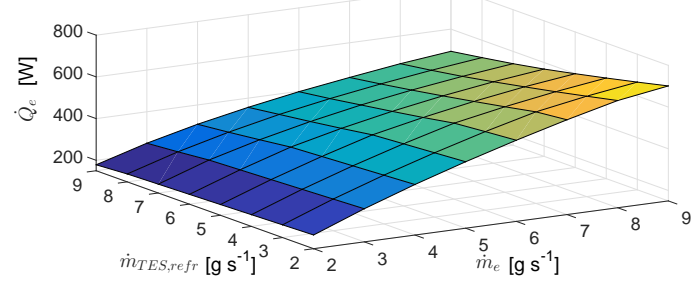

Figura 10: Relación cualitativa de $\dot{Q}_{e}$ con $\dot{m}_{e} \mathrm{y}$ $\dot{m}_{T E S, r e f r}$, modo de operación cdC.

Potencia frigorífica transferida por el refrigerante en el TES, Modo cdC

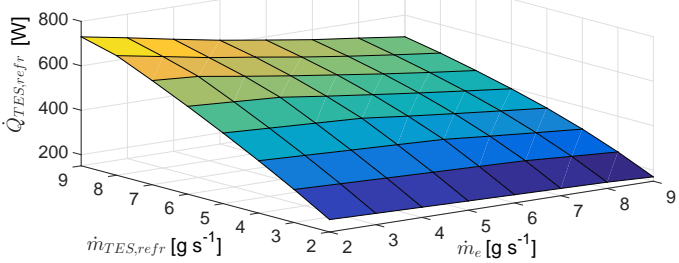

Figura 11: Relación cualitativa de $\dot{Q}_{T E S, r e f r}$ con $\dot{m}_{e} \mathrm{y} \dot{m}_{T E S, r e f r}$, modo de operación cdC.

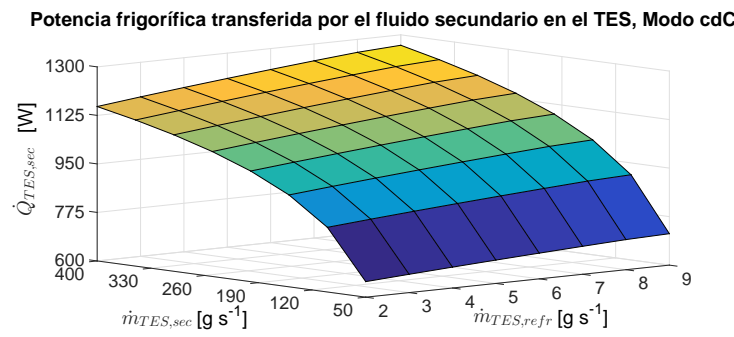

Figura 12: Relación cualitativa de $\dot{Q}_{T E S, \text { sec }}$ con $\dot{m}_{T E S, r e f r} \mathrm{y} \dot{m}_{T E S, s e c}$, modo de operación cdC. se añade un sistema de almacenamiento de energía frigorífica basado en un material de cambio de fase. Se ha descrito el modelado de cada elemento por separado y su interconexión en el simulador global. Asimismo, se ha estudiado el rango de potencias frigoríficas en los diferentes modos de operación, así como el acoplamiento entre las mismas. Se ha observado también que el grado de carga del sistema TES altera los rangos de potencia alcanzables por parte del sistema, dados unos ciertos rangos admisibles de las variables manipulables.

Estos rangos de potencia resultan clave para abordar el control del sistema. Como trabajo futuro se pretende plantear una estrategia de control en cascada donde se generen referencias para las potencias frigoríficas de acuerdo a la demanda, la predicción sobre el estado de carga del TES y la evolución horaria del precio de la energía eléctrica, mientras que un controlador de bajo nivel se encarga de manipular las variables de entrada para que el ciclo genere las potencias frigoríficas requeridas. Asimismo, se pretende validar los resultados proporcionados por el simulador tan pronto como el montaje del sistema físico finalice.

\section{Agradecimientos}

Los autores agradecen al Ministerio de Economía, Industria y Competitividad la financiación de este trabajo, mediante los proyectos DPI2015-70973-R y DPI2016-79444-R, así como a la Universidad de Sevilla mediante el VI PPIT-US.

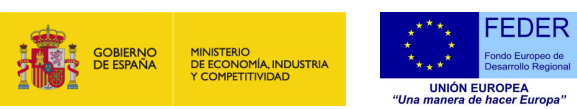




\section{English summary}

\section{SIMULATOR OF AN EXPE- RIMENTAL REFRIGERATION PLANT WITH AN ENERGY STO- RAGE SYSTEM}

\begin{abstract}
This paper describes the simulator of a vapour-compression experimental refrigeration plant which a thermal energy storage has been added to. Individual modelling of all elements is addressed, as well as their interconnection. Moreover, the cooling power ranges achieved when working in different operating modes are analysed, while the interaction between the main cooling powers is also studied.
\end{abstract}

Keywords: Refrigeration cycle, Thermal energy storage, Phase change material.

\section{Referencias}

[1] G. Bejarano, J. A. Alfaya, M. G. Ortega, and F. R. Rubio. Design, automation and control of a two-stage, two-load-demand experimental refrigeration plant. In $23^{\text {rd }}$ Mediterr. Conf. on Control and Autom., Torremolinos (Spain), pages 537-544, 2015.

[2] G. Bejarano, J. A. Alfaya, M. G. Ortega, and M. Vargas. On the difficulty of globally optimally controlling refrigeration systems. Appl. Therm. Eng., 111:1143-1157, 2017.

[3] G. Bejarano, D. Rodríguez, J. A. Alfaya, M. G. Ortega, and F. Castaño. On identifying steady-state parameters of an experimental mechanical-compression refrigeration plant. Appl. Therm. Eng., 109:318-333, 2016.

[4] G. Bejarano, J. J. Suffo, M. Vargas, and M. G. Ortega. Novel scheme for a PCM-based cold energy storage system. Design, modelling and simulation. Appl. Therm. Eng., 132:256 - 274, 2018.

[5] G. Bejarano, M. Vargas, M. G. Ortega, F. Castaño, and J. E. Normey-Rico. Efficient simulation strategy for PCM-based cold-energy storage systems. Appl. Therm. Eng., 139:419 - 431, 2018.
[6] G. Bejarano, C. Vivas, M. G. Ortega, and M. Vargas. Suboptimal hierarchical control strategy to improve energy efficiency of vapour-compression refrigeration systems. Appl. Therm. Eng., 125:165-184, 2017.

[7] I. H. Bell, J. Wronski, S. Quoilin, and V. Lemort. Pure and pseudo-pure fluid thermophysical property evaluation and the open-source thermophysical property library CoolProp. Ind. and Eng. Chem. Res., 53(6):2498-2508, 2014.

[8] T. L. Bergman, F. P. Incropera, A. S. Lavine, and D. P. Dewitt. Fundamentals of heat and mass transfer. John Wiley \& Sons, 7th edition, 2011.

[9] I. Dincer. On thermal energy storage systems and applications in buildings. Energy and Build., 34(4):377-388, 2002.

[10] I. Dincer and M. Rosen. Thermal energy storage: systems and applications. John Wiley \& Sons, 2002.

[11] M. M. MacCracken. Thermal energy storage myths. Energy Eng., 101(4):69-80, 2004.

[12] D. Rodríguez, G. Bejarano, J. A. Alfaya, M. G. Ortega, and F. Castaño. Parameter identification of a multi-stage, multi-loaddemand experimental refrigeration plant. Control Eng. Pract., 60:133-147, 2017.

[13] L. C. Schurt, C. J. L. Hermes, and A. TrofinoNeto. A model-driven multivariable controller for vapor compression refrigeration systems. Int. J. of Refrig., 32(7):1672-1682, 2009 .

[14] S. Tassou, Y. Ge, A. Hadawey, and D. Marriott. Energy consumption and conservation in food retailing. Appl. Therm. Eng., 31(23):147-156, 2011.

[15] G. Y. Yun and K. Steemers. Behavioural, physical and socio-economic factors in household cooling energy consumption. Appl. Energy, 88(6):2191-2200, 2011.

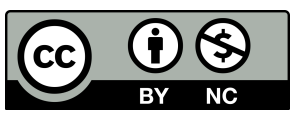
(C) 2018 by the authors. Submitted for possible open access publication under the terms and conditions of the Creative Commons Attribution CC-BY-NC 3.0 license (http://creativecommons.org/licenses/by-nc/3.0/). 